\title{
Rate of chaotic mixing in localized flows
}

\author{
Jalila Boujlel, Franck Pigeonneau, Emmanuelle Gouillart, and Pierre Jop \\ Surface du Verre et Interfaces, UMR 125 CNRS/Saint-Gobain, \\ 39, quai Lucien Lefranc, F-93303 Aubervilliers, Cedex, France
}

(Dated: September 5, 2016)

\begin{abstract}
We experimentally study the rate of chaotic mixing in viscoplastic fluids, by using a rod-stirring protocol with a rotating vessel. Only a limited zone localized around the stirring rods is highly sheared at a given time. Using a dyed spot as initial condition, we measure the decay of concentration fluctuations of dye as mixing proceeds. The mixing rate is found to be proportional to the volume of highly-sheared fluid during a rotation period of the rods, and inversely proportional to the number of rotations of the rods over a rotation of the vessel. Thanks to numerical simulations and experimental measurements, we relate the volume of highly-sheared fluid to the parameters of the flow. We propose a quantitative two-zone model for the mixing rate taking into account the geometry of the highlysheared zone, as well as the rate at which fluid is renewed inside this zone. For all experiments, the model predicts correctly the scaling of the exponential mixing rates during a first rapid stage, and a second slower one.
\end{abstract}

Chaotic advection [1] is a preferred physical mechanism for mixing fluids at low Reynolds number. The stretching and folding of fluid filaments results in an exponential separation of neighboring particles with time, and in a fast mixing rate compared to diffusion alone, as characterized for example by the decay of concentration fluctuations 2 27. Fluctuations decrease when concentration heterogeneities are stretched into thin filaments, down to an equilibrium diffusion scale at which molecular diffusion blurs filaments together [6].

An important fundamental and practical challenge consists in understanding and predicting the mixing rate from the geometrical and rheological parameters of the mixing flow. Theoretical studies [2 4, 8, 9] suggested that large-deviation statistics of the distribution of stretching factors of trajectories determine the long-time mixing rate, and numerical experiments on ideal simplified flows [4, 10, 11] confirmed the validity of such models for some cases. However, relating quantitatively kinematic flow parameters to the distribution of stretching factors, or its statistics, is hard to achieve. No such attempt has yet been made in the mixing literature for a realistic mixing flow, with the noteworthy exception of flows for which successive stretching factors are uncorrelated enough that stochastic models of random convolution account well for mixing rates as well as concentration distributions [5-7, 12]. Such cases include flow in porous media [13] or turbulent flows [5]

In this work, we consider an experimental mixing device in which the shearing and stretching of fluid particles is strongly localized around mobile obstacles, so that the distribution of stretching is simple enough that two zones can be defined: one close to the moving cylinders where the shear is high and the other part which experiences only small shear. This enables to predict analytically the mixing rate from flow parameters. For this purpose, we study the mixing of non-Newtonian viscoplastic fluids, that start to flow only when submitted to a stress larger than a critical value, called yield stress. This behavior affects the mixing performance, due to shear localization that can in the worst case generate dead zones inside the mixing device [14-16]. Since mixing yield-stress fluids is an operation involved in several industries such as cosmetic, polymer, petrochemical, pharmaceutical, or food engineering 16 18, an abundant literature in engineering [14-17, 19] has focused on design and upscaling of flows in order to reduce such dead zones. A few studies have addressed the description of chaotic advection in flows of viscoplastic [20,22] or other types [23, 24] of shear-thinning fluids, demonstrating in particular the the shear localization is often responsible for a very scattered distribution of stretching factors [21].

The main goal of this study on viscoplastic fluids is to relate the mixing rate to experimental parameters that govern it, in particular to evaluate the impact of the rheological properties of the fluid and the geometry of the device on the mixing process. To this end, we characterize experimentally the mixing rate in a two-dimensional flow with chaotic advection. We study the mixing of a transparent yield-stress fluid with a blob of the same fluid dyed with black ink (Pebeo). We use the same setup as described in 22]. The device (Fig,1]a) consists of two pairs of cylindrical stirring rods, counter-rotating with a constant angular velocity on circular trajectories. The outer cylindrical vessel is also rotating. We define the ratio $S=T_{\text {vessel }} / T_{\text {rods }}$, between the period of rotation of the vessel and the period of rotation of the rods. As yield stress fluids we use solutions of Carbopol EZ3 in water at different concentrations [25. We verified that their flow curve, i.e. the steady-state shear stress $(\tau)$ as a function of the shear rate $(\dot{\gamma})$, is well fitted by a Herschel-Bulkley model, $\tau=\tau_{c}+k \dot{\gamma}^{n}$, where $\tau_{c}$ is the yield stress and $k$ and $n$ are material parameters [26]. Since we found constant values for $n \simeq 0.33$ and $\tau_{c} / k \simeq 1$ for all polymer concentrations, in the following we describe the materials only through their yield stress value $\tau_{c}$. 

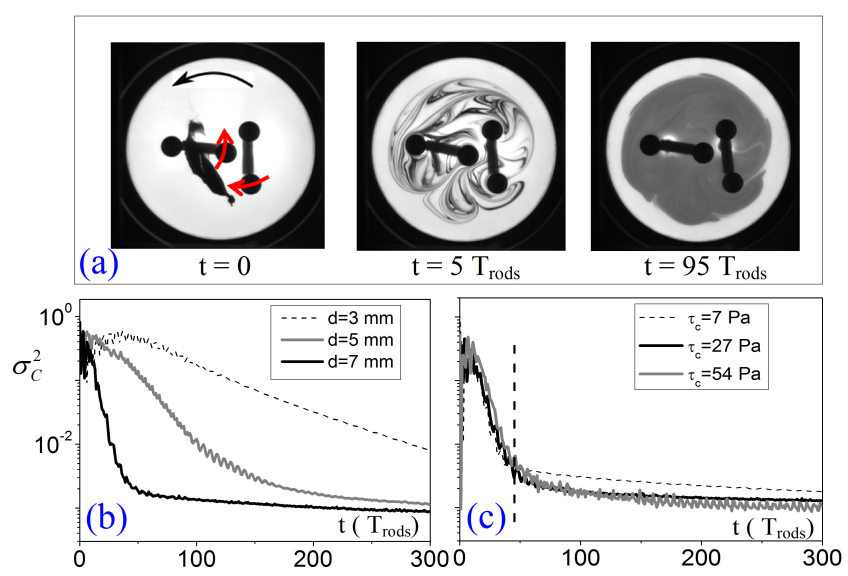

FIG. 1. (a) Typical images obtained at different times of mixing. (b) and (c) Evolution of the variance of the concentration of the dye with time, for different rod diameters $d\left(\tau_{c}=27 \mathrm{~Pa}\right)$ (b) and for different values of yield stress $\tau_{c}(d=7 \mathrm{~mm})$ (c). The vertical dashed line on graph (c) separates the two mixing regimes.

To quantify the mixing process, we follow the evolution of the dye concentration field during the experiment by taking photographs through the transparent bottom of the mixing vessel, once per rotation period of the rods. A blob of dyed fluid is released in a plane at mid-height of the vessel, inside the central zone of the vessel section (Fig 1 a left). The eggbeater-like motion of the rods as well as the rotation of the vessel promote efficient chaotic advection through stretching and folding of fluid filaments (Fig.1. a center), so that no non-chaotic islands are observed in the center of the vessel, which would have led to the formation of either dye free zone or persistent dye blob. Nevertheless, a non-chaotic dye-free zone (Fig 1 a center and right) exists close to the boundary of the vessel, consisting of fluid that is entrained in solid rotation for most of the vessel period - it is only sheared when stirring rods pass close-by. During the first periods of mixing, the initial blob of dyed fluid (Fig 1 a left) is stretched into many filaments that quickly fill the chaotic region (Fig 1 a center); the asymptotic mixing pattern (Fig.1 a right) consists of the chaotic region delineated by the dyed fluid, and the dye-free non-chaotic region.

The standard deviation of the dye concentration inside the mixing region is determined by image processing, using Beer-Lambert law for light absorption. We normalize the measured value of the standard deviation by the average value of the asymptotic concentration inside the mixing region, so that this value $\sigma_{C}$ is independent of the amount of dye injected in different experiments. The evolution of the variance with the number of rotation periods of the rods shows the existence of two distinct mixing regimes (Fig $1 \mathrm{~b}$ and c). First, exponential decay occurs when dye filaments are stretched by the rods, un-
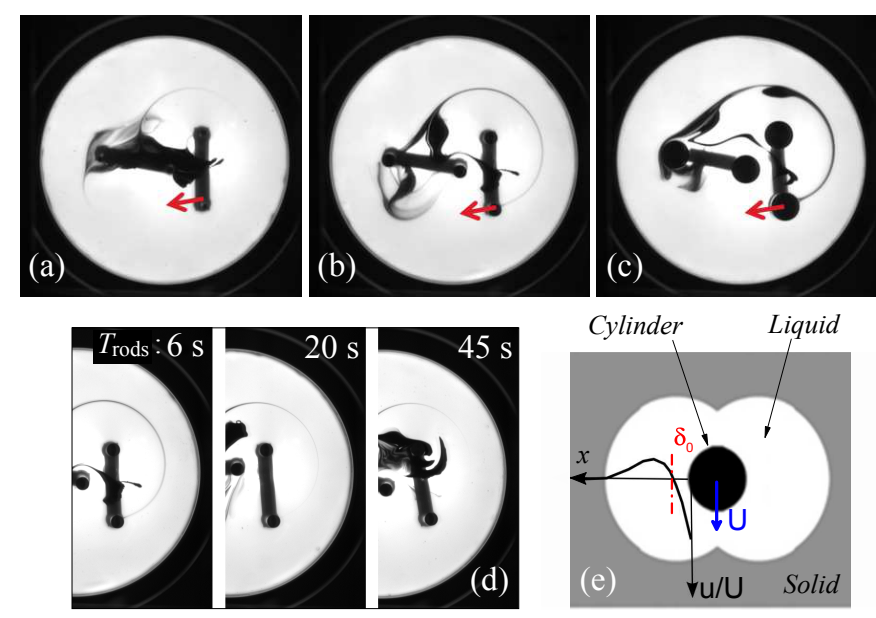

Cylinder

Liquid

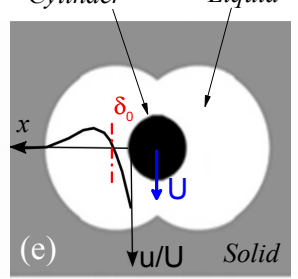

FIG. 2. (a, b, c) Pictures of mixing showing the dependence of the thickness of the dye filament formed behind the rod (red arrow), on the rod diameter: (a) $d=3 \mathrm{~mm}$,(b) $d=7$ $\mathrm{mm}$ and (c) $d=15 \mathrm{~mm}$, for $T_{\text {rods }}=6 \mathrm{~s}$. (d) Influence of the speed of the rod on the thickness of the dye filament $(d=7$ $\mathrm{mm}$ ), after the first period for $T_{\text {rods }}=6 \mathrm{~s}, T_{\text {rods }}=20 \mathrm{~s}$ and $T_{\text {rods }}=45 \mathrm{~s}$. (e) Schematic of the morphology of the flow around a cylinder moving at constant speed through a yield stress fluid, highlighting the boundary layer and the area of thickness $\delta_{0}$, where the shear is very intense and localised near the cylinder (case of low values of $\mathrm{Bi}$ ).

til their width reaches in turn the Batchelor scale [5], at which molecular diffusion balances stretching and smears out concentration fluctuations (as in Fig 1 a center). At the end of this regime, dye filaments almost fill the entire chaotic region. Then, a slower mixing regime, also exponential, occurs when remaining fluctuations are due to the slow transport of fluid from the periphery of the chaotic region (where stretching is typically lower) to the core of the chaotic region. This can be observed in Fig 1 a.right as funnels of dye-free fluid originate from the boundary of the mixing pattern, and result in filaments of fluid injected inside the mixing region with a different (lower) concentration level. In this final regime, the mixing rate is controlled by slow transport between a zone of low stretching (here, the periphery) and a zone of high stretching (the core). The resulting structured pattern of funnels of dye-free fluid being stretched into thin filaments is the so-called strange eigenmode [27, a persistent pattern that corresponds to the slowest decaying eigenmode of the advection-diffusion operator [2830. We characterize the mixing rate by the exponential rates of the first and second regimes, $\lambda_{1}$ and $\lambda_{2}$ :

$$
\sigma_{C}^{2}(t) \propto \exp \left(-\lambda_{1,2} t / T_{\text {rods }}\right) .
$$

In order to identify the mechanisms and key parameters that control the mixing process, we study the variation of the mixing rate with the diameter $d$ and the velocity of the rods $U$, the stirring ratio $S$, and the yield 
stress $\tau_{c}$ of the fluid. A first series of experiments carried out at constant $S$ shows that $\lambda_{1}$ and $\lambda_{2}$ increase significantly with the diameter (Fig, 1 b) and the velocity of the rods (described later on). However, the mixing rates do not seem to depend on the yield stress of the fluid in the range that we tested (Fig 1 c).

Flow around a rod - When a stirring rod first passes through the dyed blob, the thickness - hence the amount - of dyed fluid stretched and carried away by the rod increases significantly with the diameter of the rod (Fig]2 a, b, c.) or its speed (Fig2,d.). As we will elaborate on later, the enhanced transport and stretching of fluid result in faster mixing. Let us first estimate the volume of fluid stretched efficiently by one rod during a period of rotation. We consider the flow generated around a cylinder moving at constant velocity through a yield-stress fluid. Because of the viscoplasticity of the fluid, the flow will be strongly localized around the rods. According to the literature [31 33], there exists a limited sheared and liquid region around the cylinder, while the rest of the material is negligibly deformed and can be considered solid (Fig.2 e). Furthermore, it has been shown [33 35] for Bingham fluids [36] that the shear is very localized and very intense within a thin boundary layer close to the cylinder (see the schematic velocity profile in Fig 2 e). We note $\delta_{0}$ the typical thickness of this boundary layer. While the shear is very intense inside the boundary layer, the rest of the fluid of the liquid region is only weakly sheared. This thin boundary layer is therefore a good candidate to characterize the volume of fluid efficiently stretched by the rods.

Dimensional analysis suggests that the size of the boundary layer depends on the rod diameter $d$ and on the Bingham number $\mathrm{Bi}$, which is the ratio of yield stress to viscous stress. For a Herschel-Bulkley fluid, the Bingham number is defined as $\mathrm{Bi}=\frac{\tau_{c}}{k}\left(\frac{d}{U}\right)^{n}$. For Bingham fluids, Tokpavi et al. 35] showed numerically that $\delta_{0}$ can be expressed as $\delta_{0} \propto d \mathrm{Bi}^{-0.54}$, for a Bingham number $\mathrm{Bi}$ ranging from 10 to $2.10^{5}$. Since no information exists in the literature about the size of the boundary layer for a Herschel-Bulkley fluid, we performed numerical simulations.

To evaluated the thickness of the shear layer around a cylinder numerically, we choose the configuration of a moving 2D cylinder of diameter $d$ in a straight channel of width $w=4 d$ at constant velocity $U$. The fluid is a Herschel-Bulkley fluid whose shear stress $\sigma$ is given by $\sigma=\tau_{c}+k \dot{\gamma}^{n}$, where $\tau_{c}$ is the yield stress, $k$ and $n$ are material dependent. We set the same value for the exponent $n$ as in experiments $(n=0.33)$ and the dimensionless momentum equation simulated are scaled by the Bingham number. Numerical simulations are performed using the finite-element library Rheolef [37. Using the augmented Lagrangian method and a mixed finite-element method [38, we computed the steady velocity profile and derive the value of $\delta_{0}$ by extrapolating to zero the lateral ve- locity gradient at the wall cylinder, in the range of $\mathrm{Bi}$ $[0.003-6]$. The numerical results can be well fitted by the following law:

$$
\delta_{0}=\frac{0.038 d}{\left(0.48+\mathrm{Bi}^{1.3}\right)^{0.53}}
$$

The form of the equation is chosen to provide a good agreement of the data while keeping a compact form. The figure 3 shows the results of the simulations and the fit by equation 2 .

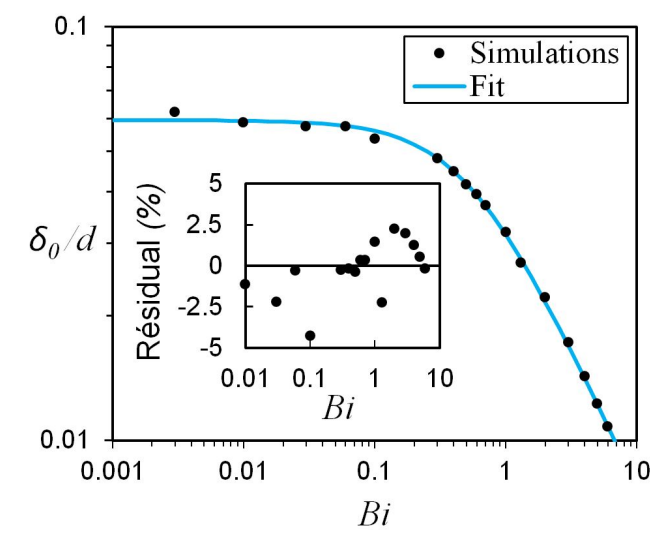

FIG. 3. Thickness $\delta$ of the strongly sheared layer around a cylinder moving in a Herschel-Bulkley fluid at constant velocity $(\bullet)$. The line is a fit following the equation 2 Inset: The residuals are lower than $2 \%$ in the range of interest of the experiemnts.

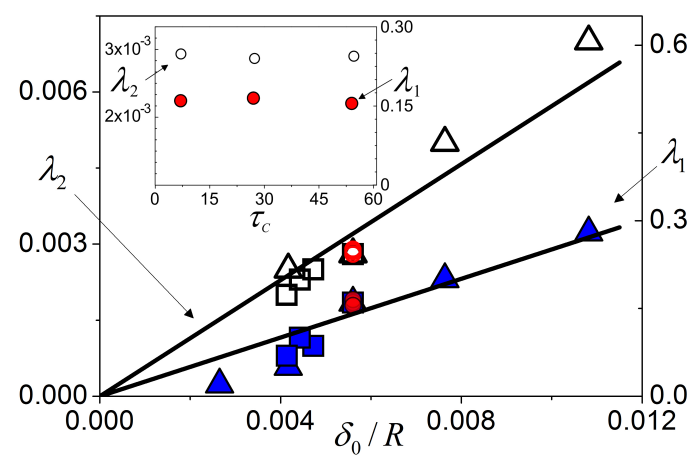

FIG. 4. Mixing rates $\lambda_{1}$ (full symbol) and $\lambda_{2}$ (empty symbol) versus $\delta_{0} / R$ (computed from Eq. 2), with $d \in\{3,5,7,10,15\}$ $\mathrm{mm}$ (triangles), $T_{\text {rods }} \in\{6,20,30,45\} \mathrm{s}$ (square) and $\tau_{c} \in$ $\{7,27,54\} \mathrm{Pa}$ (circles), and at constant stirring ratio $S=5.5$. The lines are fits forced through the origin. Inset: Same data only related to the impact of the yield stress, highlighting the constant values of $\lambda_{1}$ and $\lambda_{2}$, independent of $\tau_{c}$.

For the different experiments at constant $S$, we have represented in Fig 4 the mixing rates, $\lambda_{1}$ and $\lambda_{2}$, associated to the two mixing regimes, as a function of the 
characteristic thickness $\delta_{0}$ normalized by the radius of the area scanned by the rods $R$ (which corresponds roughly to the radius of the chaotic zone, Fig. 1. a.right). For the two regimes, all results collapse on a linear master curve, meaning that $\delta_{0}$ is the relevant parameter to capture the evolution of the mixing rates with the rod speed, the diameter, or the yield stress, and that the mixing rate of a yield-stress fluid is controlled by the boundary layer generated around the stirring rod. The good correlation between $\delta_{0}$ and the mixing rates explains the absence of impact of the yield-stress value on the mixing rate (see inset in Fig. 4). Indeed, $\delta_{0}$ depends via $B i$ on the ratio $\tau_{c} / k$, that is constant for our different fluids. Nevertheless, $\delta_{0}$ can be varied in our experiments by using different rod diameters and velocities.

We now propose a model in order to account for the linear dependence between the mixing rates and $\delta_{0}$. Let us start by considering $\lambda_{1}$, the mixing rate during the first regime. In this two-zone model, we proposed that the part of fluid highly stretched by the rods is that strongly sheared in the boundary layer. We checked this hypothesis thanks to complementary experiments where a thin strip of colored fluid was deformed by a cylinder moved at constant speed perpendicularly to the strip. Measurements obtained with different cylinder diameters show that the width of the strip associated with a deformation larger than $200 \%$ of the initial band (highly-stretched fluid portion) is proportional to $\delta_{0}$ (Fig. 5). The parameter $\delta_{0}$ is therefore a good proxy for the size of the highly sheared zone.

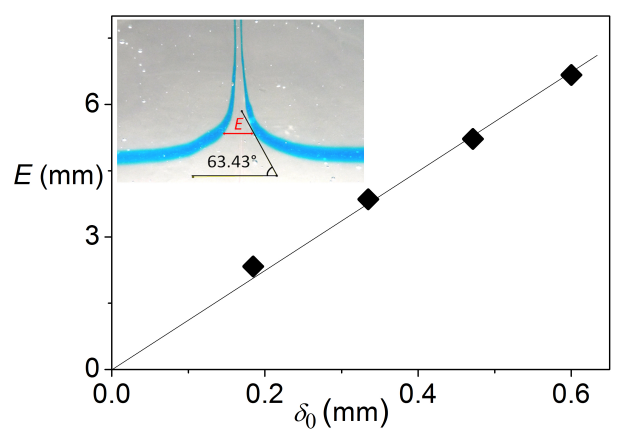

FIG. 5. Highly deformed width $E$ (deformation larger than $200 \%$ ) of an initially-straight filament by a cylindrical rod as a function of the thickness of the boundary layer for different rod radii. The rod is moving at constant speed perpendicularly to the initial filament. The line is a fit forced through the origin. Inset: The measurement of this width is done by determining the point of the colored band of fluid deformed so that its tangent lies at an angle $\theta=\arctan (2)=63^{\circ}$ relative to the initial state, which corresponds to a deformation of $200 \%$.

So, the total volume sheared during the movement of the rod over a given distance is proportional to $\delta_{0}$ times the distance travelled by the rod. Accordingly, the fraction of fluid inside the mixing area sheared during one rotation period of the rods should be proportional to $\delta_{0} / R$, and the fraction of non-sheared fluid is $\left(1-\alpha \delta_{0} / R\right) ; \alpha$ here is a constant pre-factor. If the vessel were not rotating, the rods would stretch again the same fluid particles when looping on their circular trajectory, while most of the fluid would be barely stretched for long times. However, the rotation of the vessel entrains the fluid, so that new fluid lies on the rods' trajectory when they come back. We therefore assume that the time taken for the fluid to be renewed in the highly-sheared zone on a period of rod rotation scales with $T_{\text {vessel }}$. A graphical explanation of this process is proposed in Fig. 6 (inset): the fraction of fluid renewed inside the shear zone during a rotation of the rod $\left(T_{\text {rods }}\right)$ is proportional to $1 / S$. Thus, the smaller $S$ (faster vessel), the larger the amount of new fluid sheared at each period of rotation of the rods, and the faster the mixing. We suppose that once fluid particles enter the boundary layer, their contribution to concentration fluctuations vanishes quickly; this is supported by the analysis of the filaments in Fig. 2 showing that the concentration along the stretched filaments decays exponentially and that they reach the Batchelor diffusion scale in the wake of a rod. In fact, the amount of fluid for which concentration fluctuations are erased depends on the diffusivity via the Batchelor scale. However, previous studies of scalar mixing by chaotic advection [3, 11, 3941. show that the effect of diffusivity on mixing rates induces only a weak dependency at high Peclet number. We shall therefore not consider such contribution. Under

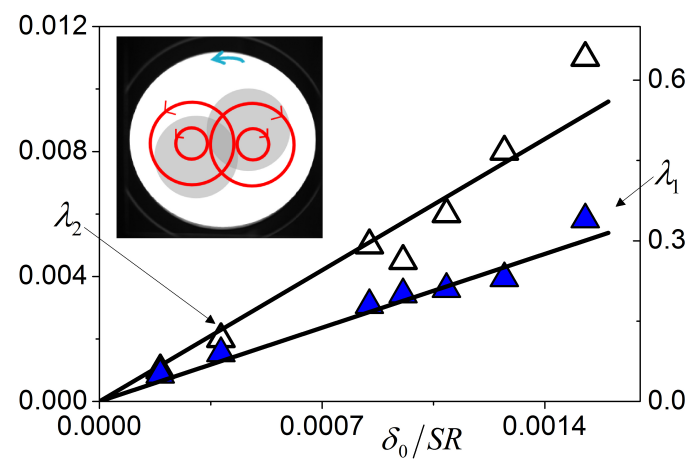

FIG. 6. Mixing rates $\lambda_{1}$ (full symbol) and $\lambda_{2}$ (empty symbol) versus $\delta_{0} / S R$ for $S$ equal to $\{5,6,7,8,9,20,40\}$. All other parameters are kept constant: $d=10 \mathrm{~mm}, T_{\text {rods }}=6 \mathrm{~s}$ and $\tau_{c}=7 \mathrm{~Pa}$. The lines are fits forced through the origin. Inset: schematic showing the impact of S: The gray area represents the envelope of the sheared zone at a time $t_{0}$, that moved with the vessel over a period $T_{\text {rods. }}$. Its longest dimension corresponds to $2 R$. At time $t=t_{0}+T_{\text {rods }}$, the zone sheared around the rods (trajectories in red) consists of part of the fluid already sheared in the previous period, and of a portion of yet unmixed fluid, that scales with $1 / S$. 
such hypotheses, the variance of the concentration can be expressed as

$$
\sigma_{C}^{2}(t) \propto\left(1-\alpha \frac{\delta_{0}}{S R}\right)^{t / T_{\text {rods }}} \approx e^{\frac{t}{T_{\text {rods }}} \log \left(1-\alpha \frac{\delta_{0}}{S R}\right)}
$$

Since the shear is strongly located close to the rods and $\delta_{0} / R$ is small, this expression is approximated as

$$
\sigma_{C}^{2}(t) \propto e^{-\alpha \frac{\delta_{0}}{S R} \frac{t}{T_{\text {rods }}}}
$$

which correctly predicts the observed linear dependency of $\lambda_{1}$ observed for the series of experiments performed at constant $S$ (Fig. 4). In order to verify the dependence on $S$, we conducted a second series of measurements where we vary only $S$, for a range $(S \geq 5)$ for which fluid is only partially renewed at each period inside the sheared zone, so that fluid particles do not leapfrog the sheared zone. The values of the mixing rates are shown in Fig 6 and confirm that $\lambda_{1}$ and $\lambda_{2}$, in our measurement range, vary linearly with $\delta_{0} / S R$. While we have developed the model for the first regime only, similar arguments can be used to account for the linear evolution of $\lambda_{2}$ with $\delta_{0} / S R$. During the second regime, most of the fluid at the core of the mixing region is well mixed and concentration fluctuations are mostly found at the periphery of the chaotic region. Therefore, the rods are most efficient at removing concentration fluctuations when their trajectory passes close to the periphery, that is, for a limited angular sector of their rotation. Within this sector, we also expect the volume of fluid that is efficiently sheared to be proportional to $\delta_{0}$, and the renewal of the fluid inside the highly sheared zone to be proportional to $1 / S$. During the remainder of the period, the rods mainly shear fluid that has already been highly stretched beforehand. The small angular sector at which the rods are the most efficient in this regime is responsible for the smaller value of $\lambda_{2}$ with respect to $\lambda_{1}$.

In conclusion, this work has shown that the scaling of mixing rates can be successfully predicted from flow parameters when shear is strongly localized. For the case of Herschel-Bulkley fluids, we have related the flow parameters to the quantity of fluid that is displaced and sheared, and hence to the mixing rates. Such quantitative understanding is to our knowledge unprecedented for chaotic advection; it is also paramount for geometry selection and upscaling in engineering. A challenge for future work consists in extending the approach to different kinds of fluid and less simplistic distributions of stretching.

The authors acknowledge the precious help of E. Garre for the experimental device, as well as support from the French ANR (project Rheomel ANR-11-JS09-015).
[1] Hassan Aref, "Stirring by chaotic advection," J. Fluid Mech. 143, 1-21 (1984).

[2] T. M. Antonsen, Z. Fan, E. Ott, and E. Garcia-Lopez, "The role of chaotic orbits in the determination of power spectra of passive scalars," Phys. Fluids 8, 3094 (1996).

[3] D. R. Fereday, P. H. Haynes, A. Wonhas, and J. C. Vassilicos, "Scalar variance decay in chaotic advection and Batchelor-regime turbulence," Phys. Rev. E 65, 035301 (2002).

[4] P. H. Haynes and J. Vanneste, "What controls the decay of passive scalars in smooth flows?" Phys. Fluids 17, 097103 (2005).

[5] E. Villermaux and J. Duplat, "Mixing as an aggregation process," Phys. Rev. Lett. 91, 184501 (2003).

[6] Jerôme Duplat and Emmanuel Villermaux, "Mixing by random stirring in confined mixtures," J. Fluid Mech. 617, 51-86 (2008).

[7] J Duplat, A Jouary, and E Villermaux, "Entanglement rules for random mixtures," Phys. rev. lett. 105, 034504 (2010).

[8] D. R. Fereday and P. H. Haynes, "Scalar decay in twodimensional chaotic advection and batchelor-regime turbulence," Phys. Fluids 16, 4359 (2004).

[9] J.-L. Thiffeault, "Scalar decay in chaotic mixing," in Transport and Mixing in Geophysical Flows, Lecture Notes in Physics, Vol. 744, edited by JeffreyB. Weiss and Antonello Provenzale (Springer Berlin Heidelberg, 2008) pp. 3-36.

[10] J. Sukhatme and R. T. Pierrehumbert, "Decay of passive scalars under the action of single scale smooth velocity fields in bounded two-dimensional domains: From non-self-similar probability distribution functions to selfsimilar eigenmodes," Phys. Rev. E 66, 056302 (2002).

[11] Y.-K. Tsang, T. M. Antonsen, Jr., and E. Ott, "Exponential decay of chaotically advected passive scalars in the zero diffusivity limit," Phys. Rev. E 71, 066301 (2005).

[12] Emmanuel Villermaux, AD Stroock, and HA Stone, "Bridging kinematics and concentration content in a chaotic micromixer," Phys. Rev. E 77, 015301 (2008).

[13] T. Le Borgne, M. Dent, and E. Villermaux, "The lamellar description of mixing in porous media," J. Fluid Mech. 770, 458-498 (2015).

[14] Paulo E Arratia, Troy Shinbrot, Mario M Alvarez, and Fernando J Muzzio, "Mixing of non-newtonian fluids in steadily forced systems," Phys. Rev. Lett. 94, 084501 (2005).

[15] Paulo E Arratia, Greg A Voth, and Jerry P Gollub, "Stretching and mixing of non-newtonian fluids in timeperiodic flows," Phys. Fluids 17, 053102 (2005).

[16] P. J. Cullen and Robin K Connelly, "Rheology and mixing," in Food Mixing, edited by P. J. Cullen (Blackwell Publishing Ltd., Oxford, UK, 2009) Chap. 4, pp. 50-72.

[17] Marko Zlokarnik, Stirring: theory and practice (WileyVCH Verlag GmbH, Weinheim, Germany, 2001).

[18] Farhad Ein-Mozaffari and Simant R Upreti, "Investigation of mixing in shear thinning fluids using computational fluid dynamics," in Computational fluid dynamics, edited by Hyoung Woo OH (InTech, Rijeka, Croatia, 2010) Chap. 4, pp. 77-102.

[19] A Amanullah, SA Hjorth, and AW Nienow, "A new 
mathematical model to predict cavern diameters in highly shear thinning, power law liquids using axial flow impellers," Chem. Eng. Sci. 53, 455-469 (1998).

[20] PE Arratia, J Kukura, J Lacombe, and FJ Muzzio, "Mixing of shear-thinning fluids with yield stress in stirred tanks," AIChE journal 52, 2310-2322 (2006).

[21] Yurun Fan, Nhan Phan-Thien, and Roger I Tanner, "Tangential flow and advective mixing of viscoplastic fluids between eccentric cylinders," J. Fluid Mech. 431, 6589 (2001).

[22] Dawn M Wendell, Franck Pigeonneau, Emmanuelle Gouillart, and Pierre Jop, "Intermittent flow in yieldstress fluids slows down chaotic mixing," Phys. Rev. E 88, 023024 (2013).

[23] Patrick D Anderson, Oleksiy S Galaktionov, Gerrit WM Peters, Frans N van de Vosse, and Han EH Meijer, "Mixing of non-newtonian fluids in time-periodic cavity flows," J. Non-Newtonian Fluid Mech. 93, 265-286 (2000).

[24] Thomas C Niederkorn and Julio M Ottino, "Chaotic mixing of shear-thinning fluids," AIChE J. 40, 1782-1793 (1994).

[25] JM Piau, "Carbopol gels: Elastoviscoplastic and slippery glasses made of individual swollen sponges:: Mesoand macroscopic properties, constitutive equations and scaling laws," J. Non-Newtonian Fluid Mech. 144, 1-29 (2007).

[26] P. Coussot, L. Tocquer, C. Lanos, and G. Ovarlez, "Macroscopic vs. local rheology of yield stress fluids," J. Non-Newtonian Fluid Mech. 158, 85-90 (2009).

[27] R. T. Pierrehumbert, "Tracer microstructure in the largeeddy dominated regime," Chaos, Solitons Fractals 4, 1091-1110 (1994).

[28] Mark A Stremler, Shane D Ross, Piyush Grover, and Pankaj Kumar, "Topological chaos and periodic braiding of almost-cyclic sets," Phys. Rev. Lett. 106, 114101 (2011).

[29] Emmanuelle Gouillart, Natalia Kuncio, Olivier Dauchot,
Bérengère Dubrulle, Stéphane Roux, and J-L Thiffeault, "Walls inhibit chaotic mixing," Phys. Rev. Lett. 99, 114501 (2007).

[30] Emmanuelle Gouillart, J-L Thiffeault, and Olivier Dauchot, "Rotation shields chaotic mixing regions from noslip walls," Phys. Rev. Lett. 104, 204502 (2010).

[31] Evan Mitsoulis, "On creeping drag flow of a viscoplastic fluid past a circular cylinder: wall effects," Chem. Eng. Sci. 59, 789-800 (2004).

[32] Nicolas Roquet and Pierre Saramito, "An adaptive finite element method for bingham fluid flows around a cylinder," Comput. Meth. Appl. Mech. Eng. 192, 3317-3341 (2003).

[33] Benjamin Deglo De Besses, Albert Magnin, and Pascal Jay, "Viscoplastic flow around a cylinder in an infinite medium," J. Non-Newtonian Fluid Mech. 115, 27-49 (2003).

[34] N Nirmalkar, RP Chhabra, and RJ Poole, "On creeping flow of a bingham plastic fluid past a square cylinder," J. Non-Newtonian Fluid Mech. 171, 17-30 (2012).

[35] Dodji Léagnon Tokpavi, Albert Magnin, and Pascal Jay, "Very slow flow of bingham viscoplastic fluid around a circular cylinder," J. Non-Newtonian Fluid Mech. 154, 65-76 (2008).

[36] For Bingham fluids, the shear stress is the sum of the yield stress and a viscous stress proportional to the shear rate.

[37] P. Saramito, Efficient C++ finite element computing with Rheolef (CNRS-CCSD ed., 2013).

[38] N. Roquet and P. Saramito, Comput. Meth. Appl. Mech. Eng. 192, 3317 (2003).

[39] Valérie Toussaint, Philippe Carrière, and Florence Raynal, "A numerical Eulerian approach to mixing by chaotic advection," Phys. Fluids 7, 2587 (1995).

[40] A. Wonhas and J. C. Vassilicos, "Mixing in fully chaotic flows," Phys. Rev. E 66, 051205 (2002).

[41] Andrew D Gilbert, "Advected fields in mapsiii. passive scalar decay in baker's maps," Dynamical Systems 21, 25-71 (2006). 